\title{
Graphene-based Reconfigurable Reflecting Surface for Future Communications
}

\author{
Salman Behboudi Amlashi, Mohsen Khalily,Tim Brown, Pei Xiao, Rahim Tafazolli \\ Department of Electrical and Electronic Engineering \\ 5GIC\&6GIC, Institute for Communication Systems (ICS), University of Surrey, Guildford, United Kingdom \\ \{s.behboudiamlashi, m.khalily, t.brown, p.xiao, r.tafazolli\}@surrey.ac.uk
}

\begin{abstract}
In this paper, a reflecting metasurface is proposed to control the reflection angle by manipulating the chemical potential $(\mathrm{CP})$ of graphene. The surface can operate in three anomalous reflection modes for $\theta=45^{\circ}, 60^{\circ}$ and $75^{\circ}$ while it is illuminated with a normal incident electromagnetic wave (EMW). Moreover, by tuning the chemical potential of graphene sheets the proposed surface can switch off the reflection mode of operation by absorbing the incident EM power.
\end{abstract}

\section{INTRODUCTION}

$\mathrm{THz}$ spectrum will become indispensable part of future wireless systems. Despite its challenges, many recent advances have asserted the pivotal roles of $\mathrm{THz}$ frequencies for next generation of communication networks. On the other hand, $\mathrm{THz}$ Metasurfaces have attracted increasing attention recently. They can control refraction and reflection by manipulating propagation of surface EMW [1]. This allows engineers to fabricate low cost and highly integrable structures for future communication systems. Generally, metasurfaces can control different characteristics of an incident electromagnetic wave such as phase, polarization and amplitude into an anomalous form, which is not possible in normal conditions. This feature of metasurfaces enables them to play a key role for next generation of applications in beyond $5 \mathrm{G}$ and $6 \mathrm{G}$ networks. Recent studies have demonstrated several potentials for metasurfaces, e.g, the improvement coverage in mobile networks for indoor and outdoor communications. Reflecting metasurfaces can control the angle of reflection by tuning the phase response of periodic scatterers, which can be achieved by different approaches based on active or passive elements [2].

\section{DESIGN}

The proposed metasurface consists of a double-layer surface overlaid on a thin layer of gold as is shown in Fig. 1(a). The structure is formed by 2D-periodic super cells in which five different unit cells are arranged in the sense that are illustrated in Fig. 1(b). The super cells are biased with various voltage lines that are connected to each graphene sheet. Each unit cell is made up of five layers, which is depicted in Fig. 1(a). The layers are formed by graphene sheets that are superimposed on the substrates of Polyimide and Si for the top layer (TL) and bottom layer (BL) respectively. The graphene sheets are etched on substrates in each layer and are shaped as a tapered triangular pattern (TTP). Anomalous reflection entails phase gradient and it can be generated by the graphene sheets with the proposed TTP. As is illustrated in Fig. 1(a), physical dimensions of the TTP graphene sheet can be manipulated for the desired phase gradient of each unit cell. It can be seen from the Fig. 1(a), in the bigger TTP, $L_{1}=860 \mu \mathrm{m}$ and $W_{1}=840 \mu \mathrm{m}$ denote length and width of each unit cell respectively. In the smaller TTP graphene, $L_{2}=470 \mu \mathrm{m}$ and $W_{2}=400 \mu \mathrm{m}$ are length and final width of the TTP respectively and $\mathrm{s}=8 \mu \mathrm{m}$ represents the primary width of it. In addition to the physical dimensions, the chemical potential of the graphene can change the surface impedance of the graphene, which consequently modifies the phase gradient of each unit cell. The designation of the unit cells follows the generalized Snell's law of reflection shown below [3]:

$$
\sin (\theta r)-\sin (\theta i)=\frac{\nabla \varphi}{k_{0}}
$$

Where, $\theta_{i}$ and $\theta_{r}$ represent the angle of the incident wave and reflected wave relative to the normal direction of the reflecting surface respectively. Moreover, $\nabla \varphi$ denotes phase gradient and $k_{0}$ is free space wavenumber. Based on the current pattern of each unit cell, changing the chemical potential of the graphene sheet cannot change the phase response of the unit cell from 0 to $2 \pi$. Hence, to produce the required phase change, a dynamic super cell is proposed instead of a dynamic unit cell. This reconfigurable metasurface is designed for a frequency of $100 \mathrm{GHz}$ as is suggested by OFCOM for future $\mathrm{THz}$ commnications [4].

\section{RESULTS}

The reflecting metasurface is designed to receive the normal incidence with $\theta=0^{\circ}$ and it can provide anomalous reflection in different directions for $45^{\circ} \leq \theta \leq 75^{\circ}$. By changing the columnar bias voltage of the graphene sheets, the reflection angle can be controlled with in a specified range. With the help of the graphene gating, a desired phase response for each unit cell is imposed. The corresponding CP for graphene sheet is calculated and listed in Table. I] Each of these CPs of the graphene sheets in top layer and bottom layer can generate desired phase response of each unit cell to support all the $2 \pi$ phase gradient. In Fig. 2,a), the phase response and the reflection amplitude of each unit cell is plotted. The results show that the average reflection efficiency is above $90 \%$ at $\theta=45^{\circ}$. To reflect into other directions, the phase response 


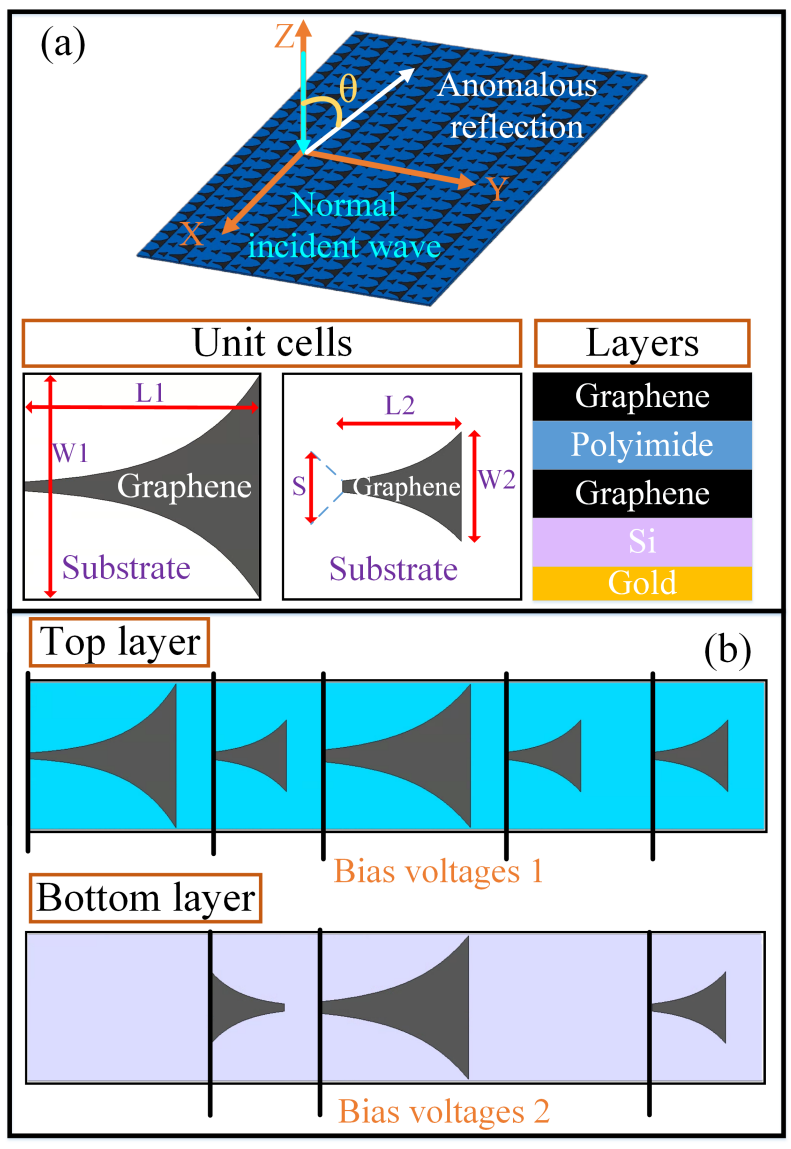

Fig. 1. The structure of the proposed reflecting metasurface.

of each unit cell and the length of the super cell $\left(5 L_{1}\right)$, which is actually fixed in this design, should be changed. In fact, the length of the super cell should be decreased while the angle of reflection, $\theta$, increases. However, There is no control over physical dimension of super cells. To work around this problem, the gating voltages for each unit cell are chosen such that all five unit cells still cover the required $2 \pi$ phase gradient but some of them are biased in a way that they have same phase response to their adjacent unit cell as calculated in Table. I Consequently, for reflections with $\theta \geq 45^{\circ}$, the efficiency is dropped to approximately $80 \%$ and $70 \%$ for $\theta=60^{\circ}$ and $\theta=75^{\circ}$ in the given order. Furthermore, with the proper gating of graphene sheets it is possible to switch off the surface. As indicated by Fig. 2(b), in switch-off mode of operation the reflection amplitude is far below the operational mode of surface. In this mode the surface operates more like an absorber instead of a reflector.

\section{CONCLusion}

A graphene-based multi-layered metasurface has been proposed that can anomalously reflect into three different directions at frequency of $100 \mathrm{GHz}$. The metasurface can be controlled by gating the graphene sheets. In addition, the surface can absorb the incident wave with proper biasing of
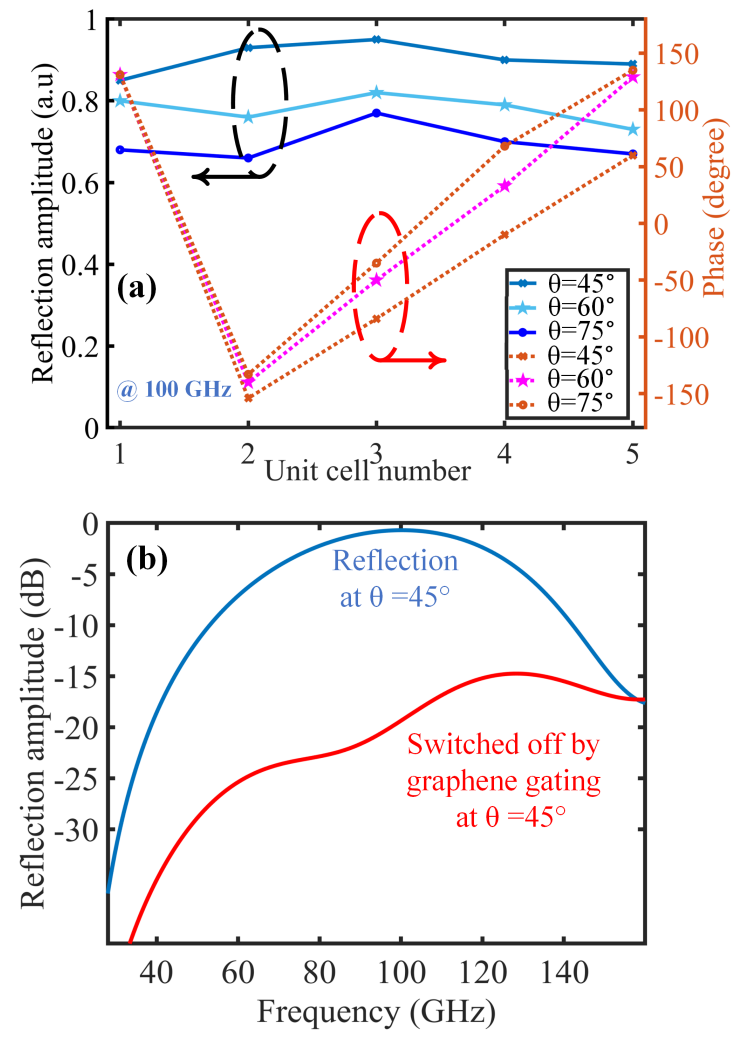

Fig. 2. Reflection wave characteristics.

TABLE I

CORRESPONDING CHEMICAL POTENTIALS VERSUS REFLECTION ANGLE.

\begin{tabular}{|c|c|c|c|c|c|}
\hline \multirow{2}{*}{ Angle } & $\begin{array}{c}\text { CP(meV) } \\
\text { cell 1 }\end{array}$ & $\begin{array}{c}\mathbf{C P}(\mathbf{m e V}) \\
\text { cell 2 }\end{array}$ & $\begin{array}{c}\mathbf{C P}(\mathbf{m e V}) \\
\text { cell 3 }\end{array}$ & $\begin{array}{c}\mathbf{C P}(\mathbf{m e V}) \\
\text { cell 4 }\end{array}$ & $\begin{array}{c}\mathbf{C P}(\mathbf{m e V}) \\
\text { cell 5 }\end{array}$ \\
\hline \multirow{2}{*}{$\theta=45^{\circ}$} & $\mathrm{TL}=250$ & $\mathrm{TL}=250$ & $\mathrm{TL}=250$ & $\mathrm{TL}=250$ & $\mathrm{TL}=250$ \\
& $\mathrm{BL}=\mathrm{NA}$ & $\mathrm{BL}=250$ & $\mathrm{BL}=250$ & $\mathrm{BL}=\mathrm{NA}$ & $\mathrm{BL}=100$ \\
\hline \multirow{2}{*}{$\theta=60^{\circ}$} & $\mathrm{TL}=250$ & $\mathrm{TL}=300$ & $\mathrm{TL}=530$ & $\mathrm{TL}=530$ & $\mathrm{TL}=250$ \\
& $\mathrm{BL}=\mathrm{NA}$ & $\mathrm{BL}=200$ & $\mathrm{BL}=200$ & $\mathrm{BL}=\mathrm{NA}$ & $\mathrm{BL}=100$ \\
\hline \multirow{2}{*}{$\theta=75^{\circ}$} & $\mathrm{TL}=250$ & $\mathrm{TL}=300$ & $\mathrm{TL}=700$ & $\mathrm{TL}=750$ & $\mathrm{TL}=530$ \\
& $\mathrm{BL}=\mathrm{NA}$ & $\mathrm{BL}=200$ & $\mathrm{BL}=200$ & $\mathrm{BL}=\mathrm{NA}$ & $\mathrm{BL}=100$ \\
\hline \multirow{2}{*}{ Switch-off } & $\mathrm{TL}=100$ & $\mathrm{TL}=100$ & $\mathrm{TL}=100$ & $\mathrm{TL}=100$ & $\mathrm{TL}=100$ \\
& $\mathrm{BL}=\mathrm{NA}$ & $\mathrm{BL}=300$ & $\mathrm{BL}=300$ & $\mathrm{BL}=\mathrm{NA}$ & $\mathrm{BL}=300$ \\
\hline
\end{tabular}

the graphene elements and operates in a switched-off mode. The efficiency of proposed surface is approximately between $90 \%, 80 \%$ and $70 \%$ for reflection angle $45^{\circ}, 60^{\circ}$ and $75^{\circ}$ respectively.

\section{REFERENCES}

[1] V. Singh, M. Khalily, S. B. Amlashi, J. D. Carey, and R. Tafazolli, "FullyTransparent transmission surface for outdoor-indoor mmwave coverage enhancement," in UCET. IEEE, Aug 2020.

[2] N. Shlezinger, G. C. Alexandropoulos, M. F. Imani, Y. C. Eldar, and D. R. Smith, "Dynamic Metasurface Antennas for 6G Extreme Massive MIMO Communications," IEEE Wireless Communications, 2021.

[3] R. Bell, K. Armstrong, C. Nichols, and R. Bradley, "Generalized laws of refraction and reflection," Optical Soc America-J, vol. 59, no. 2, pp. 187-189, feb 1969.

[4] OFCOM. Statement2021: Supporting innovation in the 100$200 \mathrm{ghz}$ range. [Online]. Available: http://www.ofcom.org.uk/ consultations-and-statements/category-2/supporting-innovation-100-200 